\title{
Outcomes of All inside ACL Reconstruction Technique in Young Patients
}

ISSN: 2576-8875

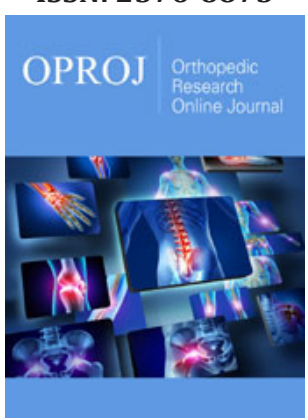

*Corresponding author: Sana Ullah, Department of Orthopedic and Spine surgery, Pakistan

Submission: 㓩April 05, 2021

Published: 眥April 20, 2021

Volume 8 - Issue 2

How to cite this article: Sana Ullah, Waqas Haleem, Muhammad Waqar, Muhammad Saeed, Israr Ahmad,d Mohammad Arif khan. Outcomes of All Inside ACL Reconstruction Technique in Young Patients. Ortho Res Online J. 8(2). OPROJ. 000682. 2021. DOI: 10.31031/OPROJ.2021.08.000682

Copyright@: Sana Ullah, This article is distributed under the terms of the Creative Commons Attribution 4.0 International License, which permits unrestricted use and redistribution provided that the original author and source are credited.
Sana Ullah*, Waqas Haleem, Muhammad Waqar, Muhammad Saeed, Israr Ahmad and Mohammad Arif khan

Department of Orthopedic and Spine Surgery, Pakistan

\begin{abstract}
The aim of this study is to assess the functional outcomes of all-inside technique for Anterior Cruciate Ligament Reconstruction (ACLR) using semitendinosus autograft, in young patients group.

Method: 40 patients were included in our study, which had anterior cruciate ligament complete tear, resulting in knee instability and pain, for which they underwent all-inside anterior cruciate ligament reconstruction, in which semitendinosus autograft was used for reconstruction, with at least 1 year of follow-up and minimum of 4 weeks of physiotherapy postoperatively.
\end{abstract}

Results: Early retune to work, load bearing along with subjective score were assessed. Mean VAS score improved from 9.2 to 1.2, mean IKDC improved from 43 to 96 while Lysholm score improved from 62 to 96 in 16 months post-surgery. Lachman test was negative in $96 \%$ of patient's postoperatively. Patients returned to work and started weight bearing fairly early (on 14th post surgery day)

Conclusion: All inside ACL reconstruction using semitendinosus autograft has good results in terms of subjective and objective outcomes in young patients. Further studies are needed to compare all inside technique with standard ACL reconstruction.

Keywords: All inside ACL reconstruction; ACL reconstruction

\section{Introduction}

Anterior cruciate ligaments have been known for almost 2,000 years and were first named by Claudius Galen of Pergamon. Anterior Cruciate Ligament (ACL) is small band inside the knee joint spanning from femur to tibia and act as internal stabilizer, preventing hyperextension, anterior tibial translation and internal tibial rotation giving knee a rotational stability. Study has shown that ACL carries mechanoreceptors that are sensitive to change in direction of movement, position of the knee joint, changes in acceleration, speed, and tension [1]. The two components of ACL are anteromedial (AM bundle) and Posterolateral (PL bundle).

ACL is the most commonly completely injured structure in knee joint with annual incidence of 1/3000, resulting in 175000 ACL injuries per year in US. A torn Anterior Cruciate Ligament (ACL) usually occurs as a result of an acute noncontact deceleration injury, forceful hyperextension, or excessive rotational forces about the knee [2-3]. Complete ACL disruption typically results in dynamic knee instability or the inability to respond to quick changes in position [4]. Anterior Cruciate Ligament (ACL) injury occurs most commonly in the patients involved in sports activity or Motor Vehicle Accidents (MVA). ACL deficient knee is found to have an increased rate of secondary meniscus injury and chondral degeneration [5-9]. There is a tenfold increase in the incidence of knee osteoarthritis after ACL tear as a natural course [10]. More than fifty percent of patients with an ACL injury will develop symptomatic osteoarthritis in the following ten to twenty years [11]. To improve knee functions and stability ACL reconstruction is gold standard surgery today. It helps to reduce progression of osteoarthritis but will not restore all knee functions as compared to uninjured knee as there are a lot of factors that play a complex role in the development of osteoarthritis changes like male gender, high BMI, time from injury to ACL reconstruction, presence of cartilage degeneration at the time of surgery and reconstruction technique [12].

Since the advent of using the arthroscope to treat Anterior Cruciate Ligament (ACL) tear, there has been a steady focus on limiting the number and extent of incisions used in ACL reconstruction surgery. Operative techniques evolved from two incisions using outside-in drilling of the femoral and tibial tunnels to an inside-out method of drilling the femoral socket, 
thereby eliminating the distal, lateral femoral incision. A relatively new minimally invasive procedure of ACL reconstruction has been developed called the all-inside technique. This technique has been described as an arthroscopic procedure "without incisions", with drilling of incomplete "blind" tibial and femoral tunnels over ACL foot- prints, preserving the external cortex for the fixation. Another important feature of the technique is the use of the semitendinosus tendon folded four times for graft preparation. Changes were made to this technique with a new generation of retractable retrograde drills, new cortical fixation systems with the possibility of increased knee stability and accurate anatomical positioning of the femoral tunnel from outside to inside, with no need for large incisions.

\section{Method}

We retrospectively reviewed our sports clinic record and recruited 40 patients (40 knees) who underwent ACL reconstruction using all inside technique and semitendanosis as autograft between March 2018 and Dec 2020 with minimum follow up for 1-year months. All the surgeries were performed by a trained orthopedic and sports surgeon and the patients were followed by one orthopedic resident. All the included patients had age between 24 to 34 years. All these patients had positive anterior Lachman test, anterior drawer test and the pivot shift test (performed under anesthesia right before surgery), also they had complete ACL tear on MRI. These patients had history of sport or non-sport related trauma to the knee with resulting ACL tear and knee instability. All the patients who underwent all inside ACL reconstruction procedure had an active lifestyle, with no vascular or neurological deficit, which were ready to participate in research study and willing to be contacted on mobile phone, had no co-morbidities and must be fit for General anesthesia and surgery. All the surgeries were performed by a single surgeon using same all inside technique for ACL reconstruction in all the patients.

Post-surgery rehabilitation: After surgery all the patients attended physiotherapy clinic for minimum of 4 weeks where they were given physiotherapy sessions and educated about weight bearing and knee kinematics.

\section{Surgical procedure}

All the surgeries were performed using spinal anesthesia. After proper scrubbing and draping, like standard ACL reconstruction technique, midway between tibial tubercle and medial boarder of tibia an incision is made for hamstring (semitendenosis) tendon harvesting. Semitendinosis tendons is identified and harvested with tendon stripper. Tendon is prepared for grafting (cleaned from muscle tissue and quadrupled) and endo button passed on both ends. Quadrupled semi T Graft width was $8 \mathrm{~mm}$ to $9.5 \mathrm{~mm}$ (average 8.5). Graft length was 6.5 to $7 \mathrm{~cm}$ mean $6.8 \mathrm{~cm}$. Flip cutter reamer was used to create retrograde, independent femoral and tibial sockets each measuring $2.5 \mathrm{~cm}$, while maintain cortices. Pulling suture is used to pass endo botton in femoral tunnel and endo button loops were used to pull the graft from anteromedial portal until the femoral socket is filled with $20 \mathrm{~mm}$ of graft. Second end button is seated on tibial side and graft is pulled in anti-grade fashion in tibial tunnel (from anteromedial tunnel into joint and then into tibial tunnel.

\section{Outcome measures}

In the follow up period after surgery the patients were assessed using VAS pain score, IKDC, Lysholm score and Lachman test along with return to work and weight bearing.

\section{Results}

1) VAS pain score: Mean pre-reconstruction VAS pain score in 38 patients out of 40 were 9.2 with loaded knee which decreased to a mean of 0.7 post-reconstruction. The remaining 2 had mean pre-reconstruction VAS pain score was 9.0 but they had a second episode of trauma post reconstruction and continued to have pain.

2) IKDC Score: Mean IKDC score increased from 43 to 90,6 weeks postoperatively.

3) Lysholm score: Prior to surgery, the mean Lysholm score was 62 while when the patients are reassessed 16 months postsurgery the score improved to a mean of 92 .

4) Lachman test: Lachman test was positive in all the patients pre-operatively while after reconstruction $96 \%$ patients had negative Lachman test at 14 and 16 month post-surgery.

5) Retune to work and weight bearing (normal gait): Patients started fairly early weight bearing and returned to work on $14^{\text {th }}$ post-surgery day.

\section{Discussion}

In athletes and young patients who have high level of functional demand ACL injury can be disabling, ACL reconstruction make the life easy and they can retune to pre-injury activity level. ACL reconstruction procedures have been passing several stages i.e., from repair to open reconstruction to arthroscopic reconstruction to all inside reconstruction technique. All inside technique for ACL reconstruction is a new procedure. Our study assessed the outcomes of all inside technique in term of subjective and objective parameters. In our study we found that all inside technique has excellent results in term of reduction in pain level and return of normal gait postoperatively. In our 38 patients out of 40, preoperative mean VAS pain score was 9.2 while the score improved to 0.7 after reconstruction, our 2 remaining patients had second episode of trauma and had continued pain. In his study James et.al [13] showed similar results (Mean VAS scores of 0.8 on the $5^{\text {th }}$ Postsurgical day). Mean IKDC score also improved from a mean of 43 to 90,6 weeks post-surgery and the patients were satisfied with return to their normal life activities which is again similar to result shown by Alexander et al. [14] in his study ( Preoperative IKDC score was 44.6-60.6 which improved to 83.8-89.7 postoperatively). Same as IKDC, Lysholm showed improvement from a mean of 62 to 92 post-reconstruction which again favors the results drawn by Alexander et al. [14] in his study ( Preoperative 53.4 while postoperative Lysholm score improved to 90-93.1). All the patients 
had positive Lachman test before surgery while after surgery $96 \%$ of the patients had negative Lachman test.

\section{Conclusion}

All inside technique for ACL reconstruction is an effective procedure for ACL reconstruction with improvement in patient subjective and objective score with retune to preinjury activity level.

\section{References}

1. The anterior cruciate ligament and functional stability.

2. Boden BP, Dean CS, Feagin JA, Garrett WE (2000) Mechanisms of anterior cruciate ligament injury. Orthopedics 23(6): 573-578.

3. Ettlinger CF, Johnson RJ, Shealy JE (1995) A method to help reduce the risk of serious knee sprains incurred in alpine skiing. Am J Sports Med 23(5): 531-537.

4. Butler D, Noyes F (1980) Ligamentous restraints to anterior-posterior drawer in the human knee. J Bone Joint Surg Am 62(2): 259-270.

5. Hawkins RJ, Misamore GW, Merritt TR (1986) Follow up of the acute non operated isolated anterior cruciate ligament tear. Am J Sports Med 14(3): 205-210.

6. Kannus P, Jarvinen M (2021) Conservatively treated tears of the anterior cruciate ligament. The Journal of Bone and Joint Surgery 69(7).
7. Mizuta H, Kubota K, Shiraishi M, Otsuka Y, Nagamoto N, et al. (1995) The conservative treatment of complete tears of the anterior cruciate ligament in skeletally immature patients. J Bone Jt Surg 77(6): 890-894.

8. Kessler MA, Behrend H, Henz S, Stutz G, Rukavina A, et al. (2008) Function, osteoarthritis and activity after ACL-rupture: 11 Years followup results of conservative versus reconstructive treatment. Knee Surgery, Sport Traumatol Arthrosc 16(5): 442-448.

9. Casteleyn PP, Handelberg F (1996) Non-operative management of anterior cruciate ligament injuries in the general population. J Bone Jt Surg 78(3): 446-451.

10. Gillquist J, Messner K (1999) Anterior cruciate ligament reconstruction and the long term incidence of gonarthrosis. Sports Medicine 27: 143156.

11. Lohmander LS, Englund M, Dahl L (2007) The long-term consequence of anterior cruciate ligament and meniscus injuries: osteoarthritis. Artic Am J Sport Med 35(10): 1756-1769.

12. Functional evaluation of anterior cruciate ligament.

13. Lubowitz J, Schwartzberg R (2015) Cortical suspensory button versus aperture interference screw fixation for knee anterior cruciate ligament soft-tissue allograft: a prospective, randomized controlled Trial. Arthroscopy: The Journal of Arthroscopic \& Related Surgery 31(9): 1733-1739.

14. Connaughton A, Geeslin A (2021) All-inside ACL reconstruction: How does it compare to standard ACL reconstruction techniques? Journal of Orthopaedics 14(2): 241-246. 